\title{
Procedimientos Aritméticos en la Resolución de Problemas de Fenómenos Variacionales
}

\section{Arithmetic Procedures in Variational Phenomena Problem-Solving}

\author{
Claudia Barajas Arenas ${ }^{*}$ \\ Sandra Evely Parada Rico ${ }^{* *}$ \\ Juan Gabriel Molina Zavaleta***
}

\begin{abstract}
Resumen
Desde el proceso matemático de elaboración, comparación y ejercitación de procedimientos, uno de los cinco procesos generales de la matemática según el MEN (1998), presentamos, en este documento, hallazgos de una investigación cuyo objetivo fue caracterizar algunas dificultades que enfrentan los estudiantes cuando resuelven problemas que implican fenómenos de variación. El estudio fenomenológico de tipo experimental permitió, a través de una metodología cualitativa, reconocer, respecto a las dificultades ligadas a procedimientos de tipo aritmético, que algunos estudiantes de nuevo ingreso a la Universidad Industrial de Santander (UIS) tienen dificultad para realizar cálculos mentales poniendo en juego relaciones y propiedades numéricas, así como emplear un sistema numérico adecuado para representar el cambio y la variación del fenómeno.
\end{abstract}

Palabras clave: Variación. Dificultades. Procesos matemáticos. Procedimientos aritméticos. Resolución de problemas.

\begin{abstract}
From the mathematical process of elaboration, comparison and practice of procedures, one of the five general mathematics processes according to MEN (1998), we presented in this document, the findings of a research which objective was to characterize some of the difficulties faced by students solving problems involving the variational phenomena. The phenomenological study of the experimental type allowed, through a qualitative methodology, to recognize, regarding the difficulties linked to arithmetic type procedures, that some students entering the Universidad Industrial de Santander (UIS) have difficulty to realize mental calculations putting at stake relations and numerical properties, as well as to use a numerical system suitable to represent the change and the variation of the phenomenon.
\end{abstract}

\footnotetext{
* Magíster en Ciencias en Matemática Educativa del Centro de Investigación en Ciencia Aplicada y Tecnología Avanzada del Instituto Politécnico Nacional de México (CICATA-IPN). Profesora del Grupo de Educación Matemática de la Universidad Industrial de Santander (Grupo EUDAMAT-UIS), Bucaramanga, Santander (Colombia). Calle 9 Carrera 27, Ciudad Universitaria, Edificio Camilo Torres, Escuela de Matemáticas Bucaramanga, Santander (Colombia), código postal 680002. E-mail: claubaren@ gmail.com.

** Doctora en Ciencias en Matemática Educativa del Centro de Investigación y de Estudios Avanzados (CINVESTAV). Institución de adscripción Universidad Industrial de Santander; Calle 9 Carrera 27, Ciudad Universitaria, Profesora de la Escuela de Matemáticas de la Universidad Industrial de Santander (UIS), Bucaramanga, Santander (Colombia). Calle 9 Carrera 27, Ciudad Universitaria, Edificio Camilo Torres, Escuela de Matemáticas Bucaramanga, Santander (Colombia), código postal 680002.E-mail: sanevepa@.uis.edu.co.

***** Magíster en Ciencias con especialidad en Matemática Educativa del Centro de Investigación y de Estudios Avanzados (CINVESTAV). Profesor Centro de Investigación en Ciencia Aplicada y Tecnología Avanzada del Instituto Politécnico Nacional de México (CICATA-IPN), Legaria, Irrigación (México). Legaria \# 694 Col. Irrigación, México D.F. C.P. 11500, código postal 11500. E-mail: jmolinaz@ipn.mx.
} 
Keywords: Variation. Difficulties. Mathematical processes. Arithmetic procedures. Resolution of problems.

\section{Introducción}

La investigación en matemática educativa se ha interesado en comprender los problemas relativos al aprendizaje (RICO, 1995) identificando dificultades emergentes de la enseñanza de la matemática escolar, las cuales están asociadas a una variedad de causas: a la complejidad de los objetos matemáticos, a los procesos de pensamiento matemático, a los procesos de enseñanza, al desarrollo cognitivo de los alumnos; a las actitudes afectivas y emocionales (ABRATE; POCHULU; VARGAS, 2006).

Viendo las dificultades desde el álgebra, Engler et al. (2004) señalan que los errores comunes, cometidos por los estudiantes, se remiten a problemas de comprensión de la aritmética ya que las dificultades que ellos presentan en el álgebra, muchas veces, no son tanto dificultades en el álgebra como problemas que se quedan sin corregir en la aritmética (ENGLER et al., 2004, p. 27). García, Serrano y Díaz (1999) afirman que la realidad cognitiva de los estudiantes para pensar e interpretar los reales muestra la profunda contradicción entre la estructura ideal e incuestionable de los reales y las nociones, ideas y concepciones de los estudiantes.

Lo anterior evidencia una problemática que resuena a nivel nacional e internacional. En el currículo nacional de Colombia (MEN, 2006) se establece que la construcción de los sistemas numéricos se realiza gradualmente, a lo largo de la escolaridad básica y media, por lo que se esperaría que los estudiantes, al ingresar a la educación superior, usen los números reales y sus propiedades con cierto dominio en diferentes situaciones.

Sin embargo, una prueba diagnóstica aplicada a 113 estudiantes de nuevo ingreso a la Universidad Industrial de Santander (UIS), en 2014, deja ver que el 34,88\% de los estudiantes tiene dificultades para aplicar procedimientos aritméticos para resolver problemas que involucran procesos infinitos, y el 40,98\% de ellos para utilizar aproximaciones numéricas o gráficas para deducir, intuitivamente, el límite de una función (BARAJAS; PARADA, 2015). La prueba diagnóstica hace parte de una de las actividades del curso de precálculo, que ofrece la UIS a estudiantes de nuevo ingreso a los programas de ingeniería y ciencias como una alternativa preventiva para atender la problemática de reprobación en el curso de Cálculo Diferencial (PARADA, 2012), y otros del área de matemáticas.

El contexto del curso de precálculo permitió a Barajas (2015) caracterizar algunas dificultades que enfrentan los estudiantes cuando resuelven problemas que implican 
fenómenos de variación, específicamente desde el proceso matemático de elaboración, comparación y ejercitación de procedimientos en el marco de una investigación fenomenológica de tipo experimental, que profundizó en los procedimientos realizados por los estudiantes a través de una hoja de procesos que acompañó a la prueba diagnóstica en el primer semestre de 2015.

De manera que, teniendo en cuenta que los procedimientos aritméticos están relacionados con el dominio del número y la estructura del sistema de numeración decimal; de las operaciones en diversos contextos; de sus propiedades y de las relaciones entre ellas (BARAJAS, 2015), en este documento se quieren dar a conocer las dificultades emergentes de la resolución de problemas asociadas a este tipo de procedimientos, esperando que ellas aporten a los profesores y a la comunidad de investigadores en matemática educativa en la comprensión de la problemática de las dificultades que aquejan el aprendizaje del Cálculo.

\section{Fundamentación teórica}

De manera sucinta reseñamos, en esta sección, cómo son entendidos los procedimientos y el proceso matemático que los integra en el currículo colombiano; asimismo, describimos un marco para aproximarnos a los procedimientos aritméticos que esgrimen los estudiantes cuando resuelven problemas que implican fenómenos de variación.

\subsection{Proceso de elaboración, comparación y ejecución de procedimientos}

La comunidad de educadores matemáticos ha venido reconociendo la importancia de promover en los estudiantes un aprendizaje que supere lo memorístico; se afirma que en el estudio de las matemáticas es necesario atender tanto a las líneas de contenidos como a los procesos (NCTM, 1989, 2003; MEN, 1998; OCDE, 2003; RICO, 2006). En ese sentido, el Ministerio de Educación de Colombia da orientaciones para la formulación de un currículo de matemáticas alineado al llamado anterior; para ello introduce, en él tres ideas claves: los procesos generales que tienen que ver con el aprendizaje matemático y que explicitan lo que significa ser matemáticamente competente; los conocimientos básicos que están organizados en cinco tipos de pensamiento matemático y el contexto que tiene ver con los ambientes que rodean al estudiante y que le dan sentido a la Matemática que aprende.

De manera particular, el reconocimiento, la percepción, la identificación y la 
caracterización de las ideas centrales del Cálculo Diferencial (variación y cambio), en diferentes contextos, están ligados al pensamiento variacional (MEN, 2006), en donde, a su vez, subyacen sus núcleos conceptuales: patrones y regularidades, procesos analíticos y análisis y funciones (SEDUCA, 2005). En tanto que los procedimientos son insertados en el proceso ECEP (proceso de elaboración y comparación de procedimientos), como lo abrevia Barajas (2015) y quien, desde el MEN (1998) y Rico (1995), da una definición de los procedimientos que supera la visión algorítmica que suele enmarcarlos: son las actuaciones, destrezas, estrategias, métodos, técnicas, usos y aplicaciones diversas que un estudiante realiza para resolver problemas de manera cada vez más hábil e independiente.

Esa definición encuentra fuerza en Rico (1995, p. 9) quien reflexiona que al limitar los procedimientos a la ejecución mecánica de tareas, "se prescinde de la invención, el ensayo, la creatividad, las conjeturas y refutaciones, la significación dentro de un contexto, y tantos otros aspectos que una visión más amplia de los procedimientos matemáticos permite contemplar".

Al respecto, las autoras Valdivé y Garbin (2013, p. 20) dicen que "las representaciones, los procedimientos y ejemplos [...] permiten conocer lo que los matemáticos usaron para resolver una situación en un cierto contexto específico". Integrando esta idea a nuestro estudio, diremos que el acercarse a los procedimientos nos lleva a tener una mirada a las ideas, imágenes, representaciones y a los contextos que los estudiantes evocan en la resolución de los distintos problemas de los objetos matemáticos del Cálculo Diferencial tomados en cuenta en los problemas de la prueba diagnóstica realizada por los estudiantes.

El proceso ECEP, desde la resolución de problemas que implican fenómenos de variación, envuelve la capacidad del estudiante para transformar procedimientos, fijando su atención en las ideas centrales del Cálculo Diferencial (cambio y variación) para establecer relaciones entre sus núcleos conceptuales (patrones y regularidades, procesos algebraicos) y efectuar nuevos procedimientos específicos que respondan al fenómeno variacional que subyace en el problema.

\subsection{Procedimientos aritméticos}

Rico (1995, p. 9) afirma que el conocimiento escolar de las matemáticas "es también conocimiento tecnológico ya que se refiere a la capacidad para aplicar unos determinados conceptos y procedimientos a la resolución práctica de problemas", entendiendo que los procedimientos son aquellas formas de actuación o ejecución de tareas matemáticas. El autor 
señala tres niveles en los procedimientos: destrezas, razonamientos y estrategias. En particular, señala que "las destrezas consisten en transformar una expresión simbólica desde una forma dada hasta otra forma, y para ello hay que ejecutar una secuencia de reglas sobre manipulación de símbolos" (RICO, 1995, p. 15), y las clasifica, según el campo de las matemáticas escolares en el que operan, en: aritméticas, métricas, geométricas, gráficas y de representación.

Los Lineamientos Curriculares de Matemáticos (MEN, 1998) de Colombia adoptan esa taxonomía para enunciar los tipos de procedimientos empleados en la resolución de problemas realizando dos ajustes; de ellos, el más importante es incluir el Cálculo Diferencial e Integral al hablar de procedimientos analíticos, en lugar de hablar de destrezas gráficas y de representación.

Los cuatro tipos de procedimientos sirven de base para la caracterización de las dificultades emergentes de la resolución de problemas de fenómenos variacionales desde la mirada del proceso ECEP. En particular, y para efectos de esta publicación, enfatizamos que los procedimientos aritméticos son aquellos relacionados con el dominio del número y la estructura del sistema de numeración decimal; de las operaciones en diversos contextos; de sus propiedades y de las relaciones entre ellas. Barajas (2015) señala, de manera a priori, unas habilidades de tipo aritmético que el estudiante debe realizar para resolver problemas de Cálculo Diferencial que requieren de este tipo de procedimiento: dominar el campo de los números reales y de las operaciones básicas y superiores; usar diferentes notaciones de los números reales y establecer relaciones para decidir sobre su uso en una situación dada.

\section{Metodología}

A continuación, indicamos las características de los participantes del estudio, el instrumento empleado para la recolección de los datos y el procedimiento de análisis de éstos.

\subsection{Participantes}

En el segundo semestre de 2014, presentaron la prueba diagnóstica 654 estudiantes admitidos al primer nivel de programas de ingenierías y de la Facultad de Ciencias de la universidad. Para seleccionar la muestra de estudio nos concentramos en los estudiantes de las carreras que reportaron menor desempeño en la prueba, es decir en 113 estudiantes que 
ingresaban a las carreras de Matemáticas, Licenciatura en Matemáticas e Ingeniería en Sistemas.

\subsection{Instrumentos}

La prueba diagnóstica (inicial y final), diseñada por la coordinación del curso de precálculo, contenía trece problemas de selección múltiple, y fue soportada por un software que arrojaba un reporte con la evaluación de cada uno de los problemas de la prueba para cada estudiante. Para tener una aproximación a los procedimientos que los estudiantes realizaban para seleccionar su respuesta, se diseñó el instrumento Hoja de Procesos que corresponde a un plegable que contenía, además de los datos de presentación, instrucción e identificación de rigor, un espacio para escribir el procedimiento elaborado para obtener una solución de cada problema.

\subsection{Análisis de datos}

Como preparación a esta etapa, la investigadora realizó un análisis a priori, así: se analizaron los problemas y se determinó que tres de ellos no serían contemplados para la investigación, porque evaluaban lo mismo que otros; los diez problemas restantes se analizaron para tener una primera idea de los posibles procedimientos que los estudiantes podrían elaborar al resolver cada problema y, a su vez, se diferenciaron los procedimientos que podrían ejecutarse en la solución. A continuación, uno de los problemas de la prueba con su análisis a priori:

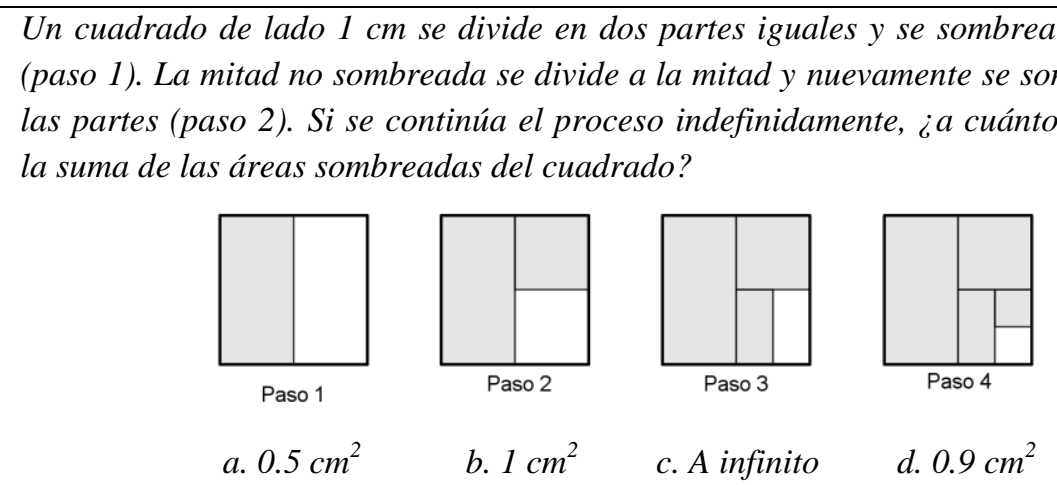

Figura 1 - Enunciado del problema del cuadrado Fuente: Barajas, 2015

Para este problema, se plantearon dos posibles soluciones que los estudiantes podrían elaborar (Figuras 2 y 3 siguientes). La primera de ellas exige distinguir el proceso infinito, 
implícito en la situación, para razonar sobre el infinito actual.

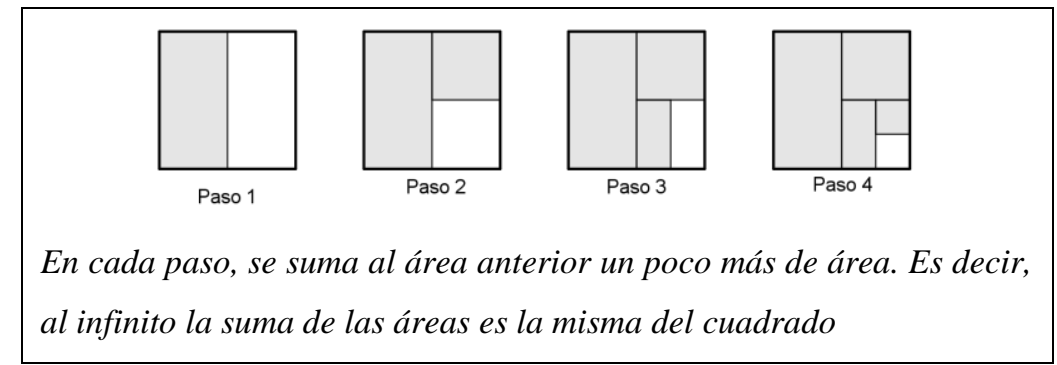

Figura 2 - Solución 1 para el problema del cuadrado Fuente: Barajas, 2015, p. 67

En la segunda solución se esperaba que algunos estudiantes elaboraran una tabla para explorar los cambios que se producen entre las variables y así manejar la noción de convergencia.

\begin{tabular}{|c|c|}
\hline PASO & ÁREA SOMBREADA \\
\hline 1 & 0,5 \\
\hline 2 & $0,5+0,25=0,75$ \\
\hline 3 & $0,5+0,25+0,125=0,875$ \\
\hline 4 & $0,5+0,25+0,125+0,0625=0,9375$ \\
\hline$\ldots$ & $\approx 1$ \\
\hline
\end{tabular}

Figura 3 - Solución 2 para el problema del cuadrado. Fuente: Barajas, 2015, p. 67

Como se puede inferir, en las soluciones hubo lugar a procedimientos aritméticos, geométricos y analíticos.

Posterior a este análisis a priori, los procedimientos de las hojas de procesos recolectadas fueron analizados y los resultados organizados en cuatro categorías que corresponden a cada uno de los tipos de procedimientos, esto a la luz del marco teórico de la investigación: dificultades en procedimientos aritméticos, dificultades en procedimientos geométricos, dificultades en procedimientos métricos y dificultades en procedimientos analíticos (BARAJAS, 2015).

A continuación, profundizamos en las dificultades en procedimientos aritméticos, tomando en cuenta el problema del cuadrado.

\section{Procedimientos aritméticos emergentes de la resolución de un problema variacional}

Para empezar, señalamos que el $22,12 \%$ de los estudiantes no elaboraron procedimiento alguno para el problema del cuadrado y, para ello, dieron justificaciones como: no sé responderlo matemáticamente; no sé cómo hacerlo ya que hace rato salí del colegio y no me acuerdo de los temas vistos; no me acuerdo de la fórmula; no me acuerdo de cómo 
resolverlo etc. (Figura 4).
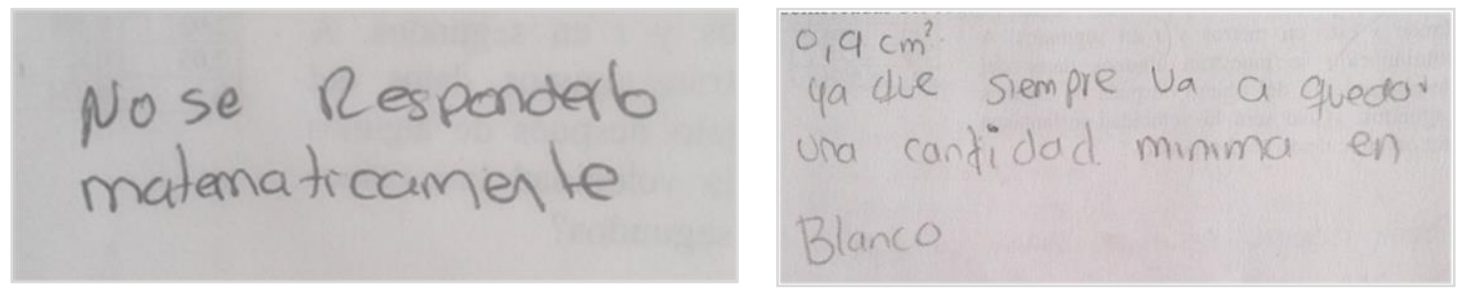

Figura 4 - Justificaciones de los estudiantes Fuente: hojas de procesos de los estudiantes

La evidencia de la derecha de la Figura 4 deja ver que otros estudiantes recurrieron a la retórica para justificar la respuesta elegida en lugar de realizar procedimientos; las justificaciones a la ausencia de procedimientos van en sentido opuesto, en términos de SantosTrigo (2007), a las habilidades de pensamiento para resolver problemas en diversos campos el cual incluye el desarrollo del pensamiento no algorítmico (aquel en el que no existe un camino determinado por seguir pero puede anticiparse), que resiste cierto nivel de incertidumbre ya que no siempre se conoce lo que se tiene al alcance en una situación o tarea. Incluso, esas expresiones reflejan la creencia de considerar que todo problema tiene una receta para ser resuelto y que si no se tiene memorizada, el problema no puede ser abordado ni se pueden diseñar estrategias para construir su solución.

El procedimiento de la Figura 5 evidencia el uso del algoritmo de la suma, dejando ver dificultades en la ejecución de la división con números decimales: para el estudiante fue correcto agregar un cero decimal tras cada nuevo paso de partición del cuadrado, acción que lo llevó a una suma cuyo resultado sería un decimal periódico infinito.

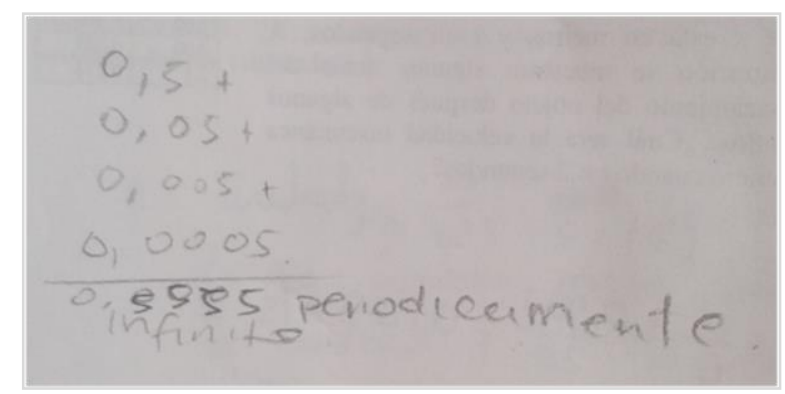

Figura 5 - Agregando ceros para suplir el algoritmo de la división Fuente: hojas de procesos de un estudiante

Podríamos interpretar que el estudiante elaboró su procedimiento de división a la luz del siguiente conocimiento heredado de los naturales: dados dos números, el que tenga más cifras es el mayor, empleándolo en este sentido: dados dos números, dividir reiteradamente un número (cinco, que es el primer dato de altura) entre el mismo número (dos, para el caso del problema) es agregar un cero decimal cada vez pues así el número es más pequeño. Este procedimiento señala que algunos estudiantes tienen arraigadas sus experiencias con los 
números naturales, pese a que en octavo grado (tres años antes de graduarse) empiezan a hablar de Números Reales. Y es que Brousseau (1989, p. 13) ya mencionaba que los números decimales se parecen tanto a los naturales que es muy fácil emplearlos, pero "esta primera comprensión se convierte en un obstáculo para un uso más refinado y para una buena comprensión de cuestiones fundamentales para el estudio de las matemáticas".

El uso de las reglas básicas y secuencias de pasos (algoritmos) ayuda a los estudiantes de matemáticas a calcular de manera más efectiva, ya que estos proporcionan un modelo, o un conjunto de directrices, para trabajar con los problemas de matemáticas (BARRINGER; POHLMAN; ROBINSON, 2010); sin embargo, cuando los estudiantes no ejecutan correctamente los algoritmos, llegan a conclusiones erradas. Esto se constata en los dos siguientes procedimientos.

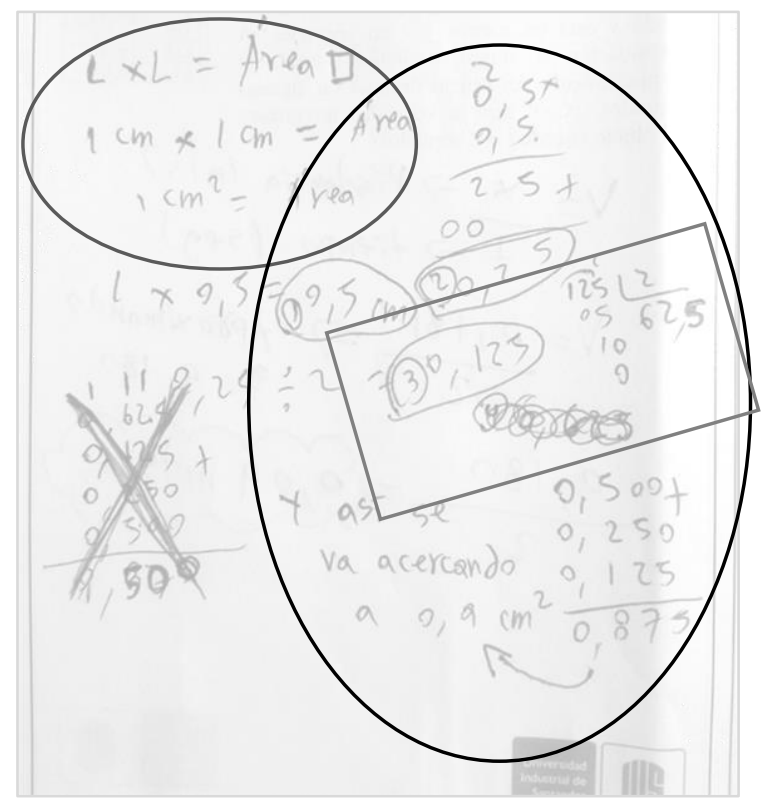

Figura 6 - Procedimientos incoherentes

Fuente: hojas de procesos de un estudiante

La Figura 6 evidencia que el estudiante empleó dos tipos de procedimientos que no conducen a la misma solución: a través del procedimiento geométrico llega a que $1 \mathrm{~cm}^{2}=$ Área (óvalo pequeño), mientras que empleando el algoritmo de la multiplicación y la división encuentra el área de la superficie sombreada para los pasos 2 y 3, y, al sumarlas, obtiene 0,875 (óvalo grande).

Del procedimiento del óvalo grande, vemos que el estudiante, sin perder de vista el proceso iterativo, razonó de la siguiente manera: al dividir el área no sombreada del cuadrado, varia la suma de las áreas, la cual es cada vez más grande en cada paso, por ello concluye su procedimiento escribiendo: y así se va acercando a $0,9 \mathrm{~cm}^{2}$. Particularmente, con los 
procedimientos señalados en el cuadrado, también se observa que el estudiante diseñó un procedimiento aritmético para evitar trabajar con la expresión decimal 0,125 para dividir y calcular el área del cuadrado en el cuarto paso: sabiendo que en el tercer paso el área es de 0,125, estableció el cociente 125:2, pero al pasar el resultado a notación decimal tuvo dificultad para escribirlo como 0,0625 ; incluso, tachó la conversión realizada y no la tomó en cuenta en la suma de las áreas del margen derecho. De manera que, de la hoja de procesos, se podría interpretar que los procedimientos son incoherentes, no obstante también vale reflexionar que tal vez la coherencia no se advierte por la hoja de procesos, que no logra capturar el razonamiento que el estudiante sigue al atender la tarea.

La dificultad para aplicar el algoritmo de la multiplicación con números decimales fue más explícita en el procedimiento de la Figura 7 que sigue.

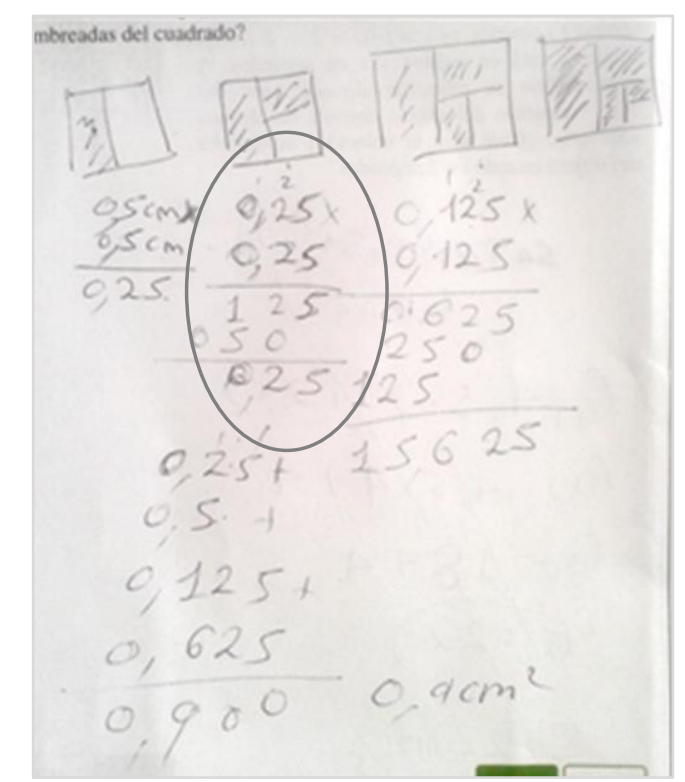

Figura 7 - Ejecución incorrecta del algoritmo de la multiplicación Fuente: hojas de procesos de un estudiante

Incluso se puede observar que el estudiante tuvo dificultad para realizar la suma de las áreas ya que, como la tiene escrita cerca al borde izquierdo, da 1,5 no $0,900 \mathrm{~cm}^{2}$. Estos procedimientos esbozan situaciones alrededor de los números decimales que son un objeto de investigación en didáctica y una fuente de problemas didácticos (BROUSSEAU, 1998). El procedimiento de la Figura 8 evidencia una representación gráfica y el uso de los fraccionarios para expresar los cuatro primeros pasos del proceso iterativo. 
Figura 8 - Uso de fracciones para plantear el procedimiento de solución Fuente: hojas de procesos de un estudiante.

Del procedimiento se puede inferir que el estudiante consideró las dos magnitudes variables del problema: el área de la superficie sombreada con las representaciones gráficas, y la suma de las áreas de las superficies pintadas (esto centrándonos en cada paso con los procedimientos aritméticos). Sin embargo, la evidencia no permite analizar cómo el estudiante pasa de $\frac{1}{2}+\frac{1}{4}+\frac{1}{8}+\frac{1}{16}$ a $\frac{120}{128}$ aunque $\frac{1}{2}+\frac{1}{4}+\frac{1}{8}+\frac{1}{16}=\frac{15}{16}$ y $\frac{120}{128}$ es equivalente a esa fracción.

Estos hallazgos permiten ver que algunos estudiantes trabajan, con preferencia, los números decimales usándolos difícilmente para acercarse al proceso infinito subyacente del problema. Y es que precisamente, la noción de infinito implícita en el fenómeno variacional del problema, causó conflictos a los estudiantes, como se aprecia en la Figura 9 siguiente.

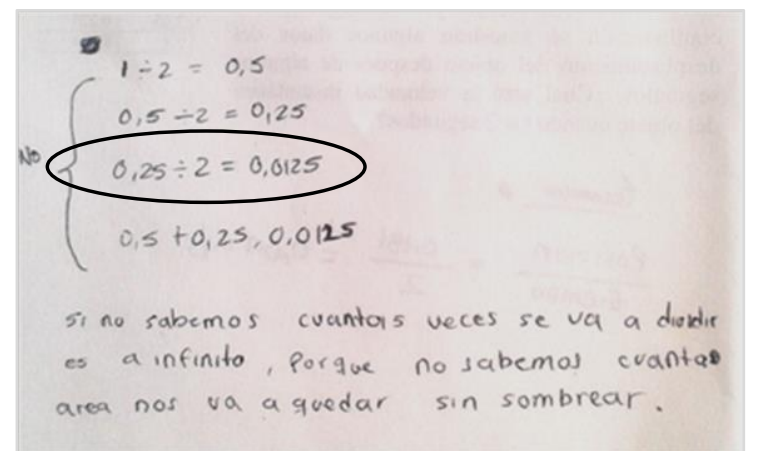

Figura 9 - Uso de fracciones para plantear el procedimiento de solución Fuente: hojas de procesos de un estudiante

Como se aprecia en el óvalo rojo de la anterior ilustración, los errores alrededor de los procedimientos con los números decimales fueron reincidentes. Errores como el señalado en el óvalo pudieron pasar desapercibidos por los estudiantes debido, interpretamos nosotros, a que en cada nuevo paso se cumplía la concepción heredada de los números naturales: al dividir un número entre otro, se obtendrán un número más pequeño lo cual no sorprende pues estudios demuestran que los estudiantes extienden las propiedades de los números naturales a los decimales, creando errores particularmente resistentes (ARTIGUE, 1990). 


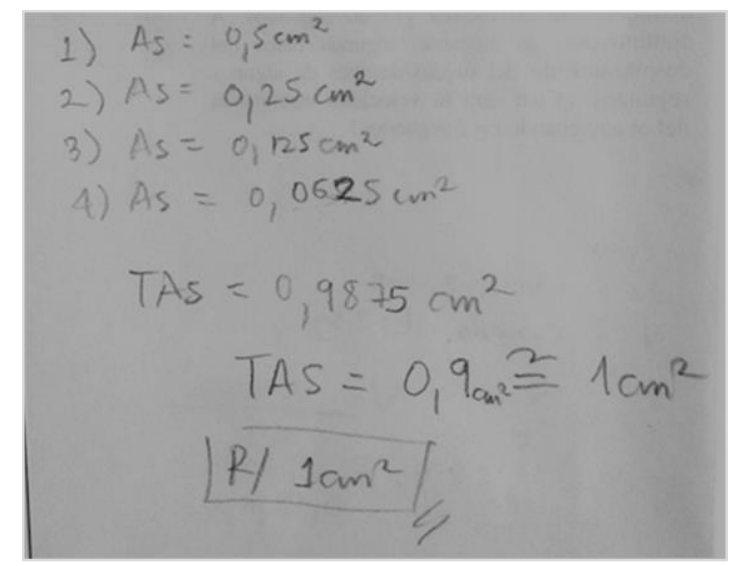

Figura 10 - Procedimientos aritméticos ligados a la noción de tendencia Fuente: Hojas de procesos de un estudiante

Pero no todo fueron errores y dificultades en la solución de este problema; en la Figura 10 anterior se puede observar que el estudiante identificó las magnitudes variables del problema y, a partir de sus habilidades aritméticas, obtuvo una aproximación a la sumatoria de las áreas para los primeros cuatro pasos, y después, con razonamientos analíticos que no son evidentes, concluyó que al continuar el proceso indefinidamente la suma de las áreas sombreadas del cuadrado se aproxima a $1 \mathrm{~cm}^{2}$.

A partir de los errores encontrados en este estudio, también podríamos deducir que los estudiantes en sus prácticas escolares empleaban la calculadora para operar con números fraccionarios (en cualquiera de sus representaciones), y por ello incurrieron en los errores señalados al trabajar a lápiz y papel, o realizando cálculos mentales en los cuales, evidentemente, no tienen mucha destreza. Esta reflexión permite rescatar que el cálculo mental requiere de la habilidad de poner en juego relaciones y propiedades numéricas pues

[...] se constituye en una práctica relevante para la construcción del sentido del sistema de numeración y las operaciones. Y se constituye en una vía de acceso para la comprensión y construcción de los algoritmos, debido a que la reflexión se centra en el significado de los cálculos intermediarios (LANZA; SCHEY, 2007, p. 8).

Resulta importante que los estudiantes, antes de ingresar a la universidad, usen las propiedades de los números reales para establecer relaciones entre ellos y las propiedades y, así, cuando empleen algoritmos puedan tener el control sobre éstos. O, como se evidencia en la Figura 11, estar en capacidad de determinar si es necesario realizar cálculos o dejarlos expresados para identificar patrones y regularidades que, posteriormente, permitan analizar la tendencia de la serie asociada al problema. 


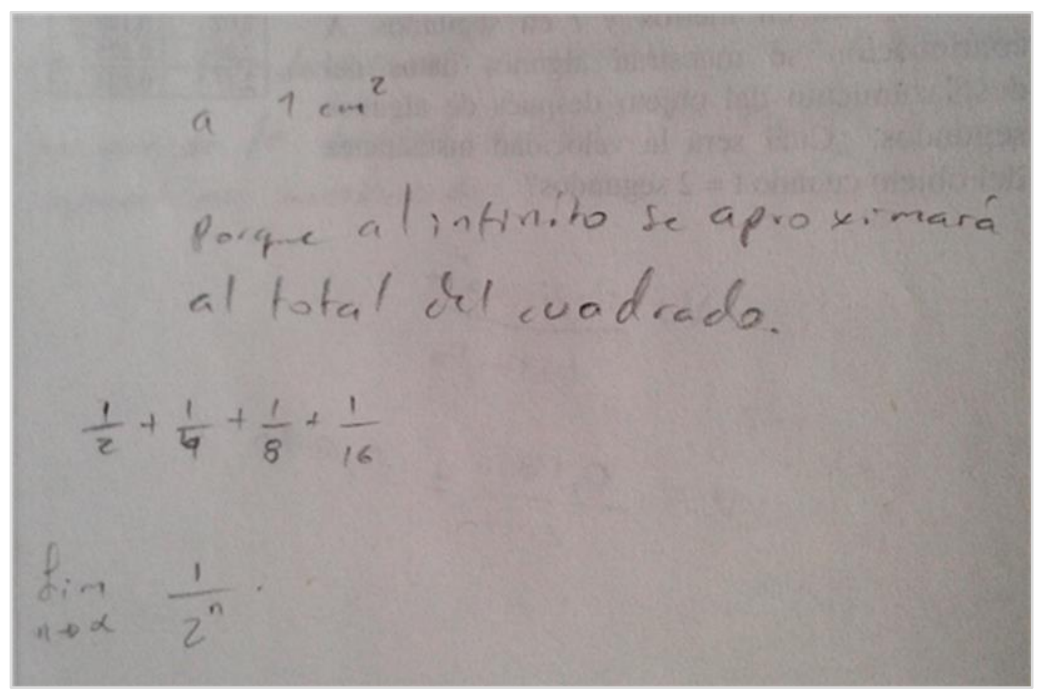

Figura 11 - Razonamiento analítico del problema

Fuente: hojas de procesos de un estudiante

Aunque son distintas las observaciones que se podrían realizar de la Figura 11 en términos de otro tipo de procedimientos, diremos que el estudiante elaboró un procedimiento aritmético (con racionales) para expresar el área sombreada hasta el cuarto paso ( $n$ ) y construyó un límite para cuando $n \rightarrow \infty$ empleando el término $n$-ésimo de la serie. El estudiante no tuvo dificultad para definir una sucesión como una función cuyo dominio son los números enteros positivos que representan los pasos de la partición. Lo declarado por el estudiante también podría reflejar que está considerando la representación gráfica dada, pues si observamos la figura del problema, ésta se va rellenando de color a medida que los pasos aumentan, entonces el área será $1 \mathrm{~cm}^{2}$, tal cual lo consideró un estudiante (ver Figura 12).

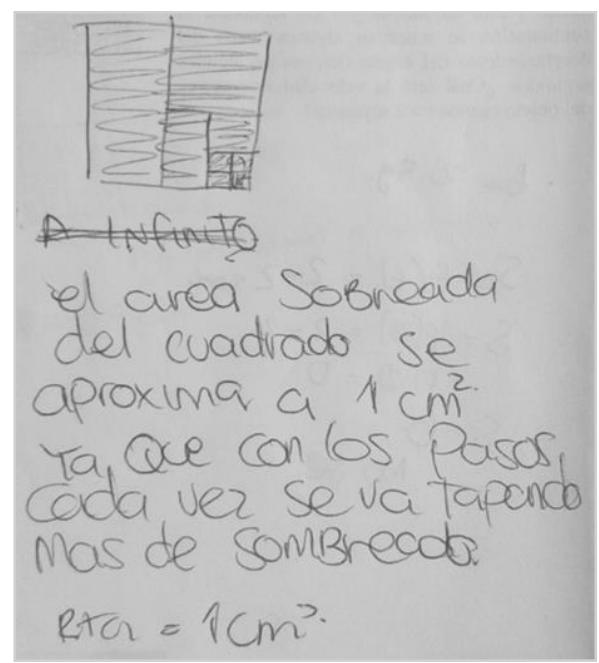

Figura 12 - Solución correcta sin procedimiento

Fuente: hojas de procesos de un estudiante

La Figura 12 nos permite precisar que los anteriores procedimientos son ejemplos representativos del $48,67 \%$ de los estudiantes que elaboraron procedimientos aritméticos para 
dar una solución a este problema (el procedimiento de la Figura 12, por ejemplo, no es considerado aritmético, ya que la evidencia refleja un análisis sobre la tendencia del área sombreada, aspecto que permite categorizarlo en otra naturaleza de procedimiento). Asimismo, los anteriores resultados dejan ver que las características de los recursos, el nivel de desarrollo de los procesos matemáticos y el conocimiento conceptual de los estudiantes para solucionar problemas dan apertura a una variedad de procedimientos para solucionar un problema, procedimientos que dan cuenta (en mayor o menor grado) de sus fortalezas y dificultades en los componentes mencionados.

\section{Conclusiones}

En Colombia, cerca de uno de cada dos estudiantes que ingresa a la educación superior no se gradúa (MEN, 2010); uno de cada cinco se retira en primer semestre o emigra hacia otras áreas. Esta problemática ha conducido a las universidades a la creación de distintas alternativas preventivas, como los son los cursos de precálculo. Otra estrategia, emergente de la preocupación sobre este tema, es la búsqueda de claridad sobre las dificultades de las matemáticas que aquejan a los estudiantes a través de estudios como el que aquí se reportó, el cual intentó responder a la pregunta de investigación ¿con cuáles dificultades llegan los estudiantes a la universidad para resolver problemas que implican situaciones de variación, específicamente desde el proceso matemático de elaboración, comparación y ejercitación de procedimientos?

En la búsqueda por aportar evidencia concreta de las dificultades de los estudiantes alrededor de la matemática, y al profundizar en la elaboración y la ejecución de procedimientos aritméticos a través de las hojas de procesos, encontramos que para resolver el problema del cuadrado, algunos estudiantes de nuevo ingreso a la UIS tienen dificultades, específicamente, para establecer relaciones y diferencias entre diferentes notaciones de números reales para decidir sobre su uso en una situación dada, realizar cálculos mentales poniendo en juego relaciones y propiedades numéricas, seleccionar adecuadas representaciones de los números para representar el cambio y la variación del fenómeno, además de reconocer el efecto de una representación u otra en la resolución de un problema (en particular para identificar una regularidad que lleve a una generalización y, a la vez, a analizar una tendencia en los datos).

Vale la pena reflexionar sobre el $22,12 \%$ de los estudiantes que no solucionaron el 
problema, dando ciertos argumentos en lugar de una posible solución. Tal vez esos argumentos son un reflejo de la actitud hacia la matemática que se han formado estos estudiantes en la escuela, una actitud que parece contraria a lo que el MEN desea promover, pues ellos no tienen una actitud positiva, con la mente abierta para afrontar un reto matemático y explorar el problema para encontrar la solución, no intentan examinar y se dan por vencidos sin intentarlo. Quisieran responder con cosas ya vistas que no recuerdan, con procedimientos escolares, no creen que ellos podrían proponer una solución. Se hace evidente que se deben sugerir estrategias para cambiar esta actitud de los estudiantes ante situaciones matemáticas nuevas para ellos.

Como reflexión final, nos queda mencionar que este trabajo más que concretar, desde el proceso de elaboración, comparación y ejecución de procedimientos, las dificultades a las cuales se enfrentan los estudiantes cuando resuelven problemas que implican fenómenos variacionales, es una invitación para que la comunidad de educación matemática centre esfuerzos en indagar, con mayor profundidad, en la Didáctica del Cálculo Diferencial desde la resolución de problemas ya que, aunque nosotros aunamos esfuerzos por rescatar los hallazgos importantes sobre dificultades alrededor de los procedimientos aritméticos, sabemos que el método empleado para caracterizarlas presenta algunas limitaciones. La más importante de ellas es que no permite saber con precisión lo que el estudiante pensaba cuando resolvía el problema, aspecto que deja abierta la puerta para estudiar con más fineza la problemática. Lo que sí, es claro, es que los conceptos y procedimientos asociados a la aritmética que los estudiantes han adquirido en el colegio requieren fortalecerse para restablecer la conexión entre ellos y los núcleos conceptuales del Cálculo (cambio y variación) de manera que se favorezcan sus competencias matemáticas en la resolución de problemas propios de esta rama de la Matemática.

\section{Referencias}

ABRATE, R.; POCHULU, M.; VARGAS, J. Errores y Dificultades en Matemática. Análisis de causas y sugerencias de trabajo. 1a. ed. Buenos Aires: Universidad Nacional de Villa María, 2006. Disponible en: 〈http://unvm.galeon.com/Libro1.pdf〉. Acceso en: 16 jul. 2014.

ARTIGUE, M. Epistémologie et didactique. Recherches en didactique des Mathématiques. 10 (2,3), 1990. pp. 241-286. In: GODINO, J. (Ed.) Marcos teóricos de referencia sobre la cognición matemática. 2003. Departamento de Didáctica de la Matemática [en línea], disponible en: http://www.ugr.es/ jgodino/fundamentos-teoricos/02 MarcosCM.pdf>. Acceso en: 13 oct. 2014.

BARAJAS, C. Dificultades del pensamiento variacional: una mirada al proceso elaboración, comparación y ejercitación de procedimientos. 2015. 185f. Tesis de maestría no publicada (Magister en Ciencias Especialidad en Matemática Educativa) - CICATA-IPN, México, 2015. 
BARAJAS, C.; PARADA, S. Una mirada al proceso matemático de elaboración, comparación y ejercitación de procedimientos en la resolución de problemas con el que ingresan los estudiantes a la universidad. In: CONFERENCIA INTERAMERICANA DE EDUCACIÓN MATEMÁTICA, 14., 2015, Tuxtla, México. Anais...Tuxtla: Patrick (Rick) Scott y Ángel Ruiz, 2015. Disponible en: <http://xiv.ciaem-redumate.org/index.php/xiv_ciaem/xiv_ciaem/paper/viewFile/612/273 >. Acceso en: 20 jun. 2015.

BARRINGER, M.D.; POHLMAN, C.; ROBINSON, M. Schools for All Kinds of Minds: Boosting Student Success by Embracing Learning Variation. ${ }^{\mathrm{a}}$. ed. San Francisco: Jossy-Bass, 2010. Disponible en: $<$ http://www.allkindsofminds.org/thinking-with-numbers-learning-and-using-mathprocedures $>$. Acceso en: 15 nov. 2014.

BROUSSEAU, G. Les obstacles épistémologiques et la didactique des mathématiques. Construction des savoirs. In: BEDNARZ, N.; GARNIER, C. (Ed.). Construction des savoirs: Obstacles et conflicts. Montréal, Canada: Agence d'Arc. Ottawa: CIRADE, 1989. p. 41-63.

BROUSSEAU, G. Théorie des Situations Didactiques. 1ª ed. Grenoble: La Pensée Sauvage, 1998.

ENGLER, A. et al. Los errores en el aprendizaje de matemática. Revista Premisa, Argentina, v. 6, n. 23, p. 23-32, 2004. Disponible en: 〈http://www.soarem.org.ar/Documentos/23\%20Engler.pdf>. Acceso en: 10 may. 2014.

GARCÍA, G.; SERRANO, C.; DÍAZ, H. ¿Qué hay detrás de las dificultades que presentan comprensión del concepto del número real? Revista Tecne, Episteme Y Didaxis, Colombia, v.5, p. 216, 1999. Disponible en: 〈http://www.pedagogica.edu.co/storage/ted/articulos/ted05_03arti.pdf >. Acceso en: 9 jul. 2014.

LANZA, P.; SCHEY, I. Todos pueden aprender (Matemática en $3^{\circ}$ ). 1a. ed. Argentina: Fondo de las Naciones Unidas para la Infancia y Asociación Civil Educación para Todos, 2007. Disponible en: < http://www.unicef.org/argentina/spanish/Mate_3ro_web.pdf >. Acceso en: 15 ago. 2014.

MINISTERIO DE EDUCACIÓN NACIONAL. Lineamientos curriculares en matemáticas. Bogotá: Autor, 1998.

. Estándares básicos de competencias en Matemáticas. Bogotá: Autor, 2006.

. Ingreso, permanencia y graduación. Educación superior. Boletín informativo, $n^{\circ} 14$, febrero de 2010.

NATIONAL COUNCIL OF TEACHERS OF MATHEMATICS (NCTM). Evaluation: Standard 9 Mathematical Procedures, 1989. [Versión en línea]. Disponible en: <http://www.fayar.net/east/teacher.web/math/standards/previous/CurrEvStds/evals9.htm>. Acceso en: 16 de febrero de 2014.

. Principios y Estándares para la Educación Matemática. Traducción de M. Fernández (Traducción de la versión del 2000 del NCTM). 1a. ed. Sevilla: SAEM Thales, 2003.

ORGANIZACIÓN PARA LA COOPERACIÓN Y DESARROLLO ECONÓMICO (OCDE). Marcos y pruebas de evaluación de PISA 2012 Matemáticas, Lectura y Ciencias, 2003. [En línea]. Disponible en: <http://www.pisa.oecd.org>. Acceso en: 20 abr. 2014.

PARADA, S. Una estructura curricular para atender la problemática relacionada con el curso de Cálculo I en la Universidad Industrial de Santander. Documento interno no publicado de la Escuela de Matemáticas. Bucaramanga, Colombia: UIS, 2012. 
RICO, L. Errores y dificultades en el aprendizaje de las matemáticas. In: LILPATRICK, J.; GÓMEZ, P.; RICO, L. (Ed.). Educación Matemática. Errores y dificultades de los estudiantes. Resolución de problemas. Evaluación. Historia. México: Grupo Editorial Iberoamérica, S.A. de C.V, 1995. p. 69108.

. La competencia matemática en PISA, PNA, v. 1, n. 2, p. 47-66, 2006. Disponible en: $\langle$ http://funes.uniandes.edu.co/529/1/RicoL07-2777.PDF〉. Acceso en: 11 may. 2014.

SANTOS-TRIGO, L. M. La resolución de problemas matemáticos. Fundamentos cognitivos. 1a. ed. México: Trillas, 2007.

SECRETARÍA DE EDUCACIÓN PARA LA CULTURA DE ANTIOQUIA (SEDUCA). Interpretación e Implementación de los Estándares Básicos de Matemáticas. Colombia: Gobernación de Antioquia, 2005.

VALDIVÉ, C.; GARBIN, S. “¿Cómo piensan los estudiantes el infinitesimal antes de iniciar un curso de análisis matemático?” Paradigma, Venezuela, v. XXXIV, n .1, p. 117-144, jun. 2013.

Submetido em 21 de Maio de 2017. Aprovado em 07 de Novembro de 2017. 